\title{
NUMERICAL MODELLING OF SLOPE STABILITY IN OPEN PIT LIGNITE MINES: A COMPARATIVE STUDY
}

\author{
Deliveris A.V. ${ }^{1}$, Zevgolis I.E. ${ }^{2}$ and Koukouzas N.C. ${ }^{1}$ \\ ${ }^{1}$ Centre for Research \& Technology Hellas - Chemical Process \& Energy Resources Institute \\ (CERTH - CPERI), Egialias 52, Marousi, 15125, Greece, deliveris@certh.gr, \\ koukouzas@certh.gr \\ ${ }^{2}$ Democritus University of Thrace, Department of Civil Engineering, Vas. Sofias 12, Xanthi, \\ 67132,Greece,zevgolis@civil.duth.gr
}

\begin{abstract}
Slope stability of an open pit lignite mine was studied using numerical methods (FEM and FDM), by means of three different software packages whose performance was compared and similarities - differences were identified. Plane strain analyses were performed using the shear strength reduction technique under drained conditions and Mohr-Coulomb constitutive models. Based on the results, all three programs demonstrated qualitatively a good agreement in the determination of safety factors and the kinetics of the collapse mechanisms. Small differences were observed in terms of shear strains localizations. Important differences were evident in the development of plastic (shear) and tensile failure indicators. Nevertheless, the problem under examination may be addressed satisfactorily by all three programs.
\end{abstract}

Keywords: geotechnical engineering, shear strength, finite elements, finite difference, limit equilibrium.

\section{Пєрі́ $\eta \psi \eta$}

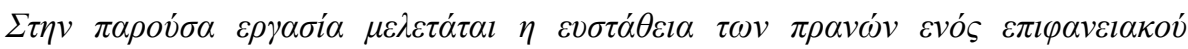

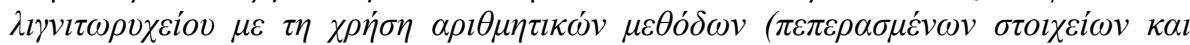

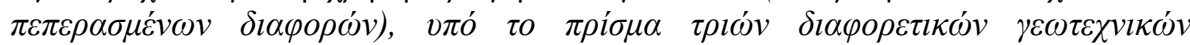

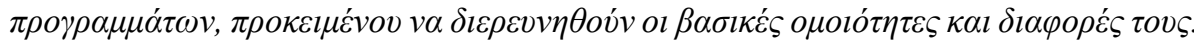

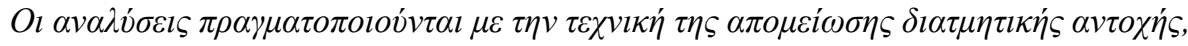

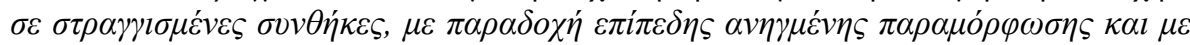

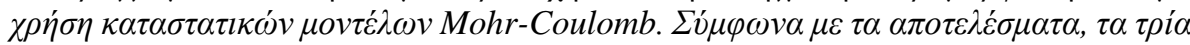

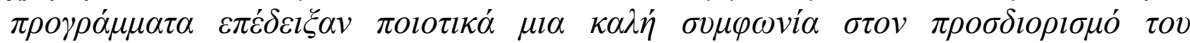

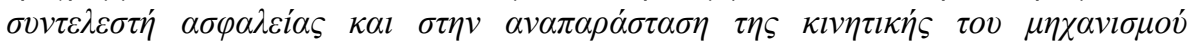

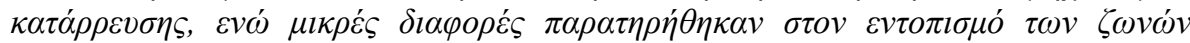

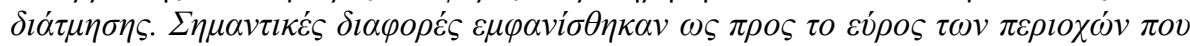

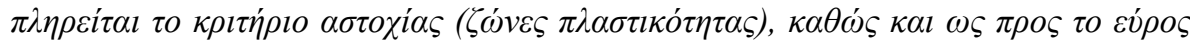

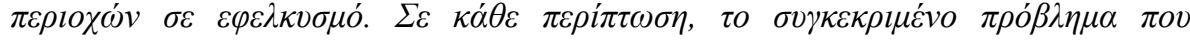

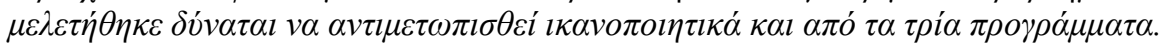

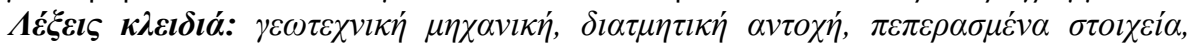

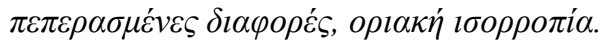




\section{Introduction}

Slope design of open pit mine is a trade-off between stability and economics (Steffen et al., 2008). The steeper the slope the less expensive it is to construct, as steeper slopes result in the reduction of the stripping ratio, i.e. the ratio of waste to ore. However, on the other hand, the flatter the slope the more stable it is. Therefore, the aim of any open pit mine design is to provide an optimal excavation configuration in the context of safety, ore recovery and financial return (Read and Stacey, 2009). Based on the above, geotechnical engineering comprises an important part of any credible open pit mine design. Due to the potentially hazardous nature of open pit mines, the application of sound geotechnical practice to their design is required, in order to allow for safe and economic mining of any commodity within ground.

Limit equilibrium methods have served geotechnical engineering, and particularly slope stability problems for many decades. Through force and/or moment equilibrium, these methods calculate a safety factor (SF), which is defined, with respect to the shear strength of the soil, as the ratio of the available shear strength to the shear stress at equilibrium (Duncan and Wright, 2005). Their basic characteristic is the simplicity and their proven, through decades of use, validity for relatively simple geometries and conditions. However, stability problems in large-scale open pit mines often involve complexities that are not easily addressed by limit equilibrium methods. In this case, using (the more sophisticated and powerful) numerical methods, such as the finite element method (FEM) and the finite difference method (FDM), provides the engineer with the opportunity to conduct more comprehensive and sound slope stability analyses. The main advantage of numerical methods is that no assumption needs to be made about the shape, direction and location of slip surfaces: failure occurs "naturally" through zones on which soil's shear strength is unable to sustain the applied shear stresses (Duncan, 1996; Griffiths and Lane, 1999). Additionally, numerical models are able to generate stress - strain distributions (deformational response), which may be of crucial importance for a robust interpretation of slope behavior, and also to address and analyze precisely complicated geometries, simulate pit excavation stages and their effect on the stress state, address possible groundwater seepage, etc. On the other hand, it is important that engineers understand not only slope stability principles, but also the limitations of the methods, before using sophisticated computer programs (provoked by their recent, extremely high, user-friendliness) and especially before interpreting and apply the results through design (Kliche, 2011). Particularly in the context of largescale mining slopes, obtaining realistic input information and interpreting the results are the most difficult aspects of numerical modelling (Hustrulid et al., 2001).

The objective of the present work is to investigate the comparative performance of three different software packages, in the context of slope stability of an open pit lignite mine. More specifically, the widely used geotechnical software FLAC 2D of Itasca Consulting Group Inc., PLAXIS 2D of Plaxis bv and Phase 2 of Rocscience Inc. are used by applying the so-called shear strength reduction technique. The scope of the work is to compare the solutions obtained for the exact same problem from the three software packages and to discuss the comparative similarities and differences among them. The paper starts with the problem statement and the definitions and assumptions of the baseline model. It continues with the presentation of the results, in terms of slip surfaces, kinetics of collapse mechanisms, and failure extents. These issues are addressed by means of discussion on shear strain localization, displacement vectors distributions and plastic regions, respectively. The paper ends with the conclusions that were drawn from the work and some recommendations for future research.

\section{Problem Statement and Numerical Model}

According to Euracoal (2013), Greece is constantly placed among the three leading lignite producers in the EU-28. In fact, domestic lignite - with a share of about $60 \%$ in power generation and accounting about $30 \%$ of primary energy consumption - is the most important indigenous fuel of Greece (Koukouzas and Koukouzas, 2000). In 2012, 62.2 Mt of lignite were mined in Greece and 
257.2 $\mathrm{Mm}^{3}$ of overburden and interburden material were excavated (Euracoal, 2013). This huge earth-moving process has been a constant challenge from a geotechnical point of view (Leonardos and Terezopoulos, 2003; Agioutantis et al., 2006; Steiakakis and Agioutantis, 2010; Kavvadas et al., 2013; Zevgolis et al., 2015).

In the present work, a typical cross section of a private open pit lignite mine in northern Greece, excavated with non-continuous extracting methods (excavators with trucks), has been used as the baseline case study. Boundaries, geometric conditions and lithologic features (geologic materials with their respective geotechnical properties) of the examined problem are all shown in Figure 1 and have been used as such in the following numerical analyses. As shown in the figure, the major lignite seam is located between and in parallel to the overburden marl/lignite intercalations layer and the underlying marl bedrock.

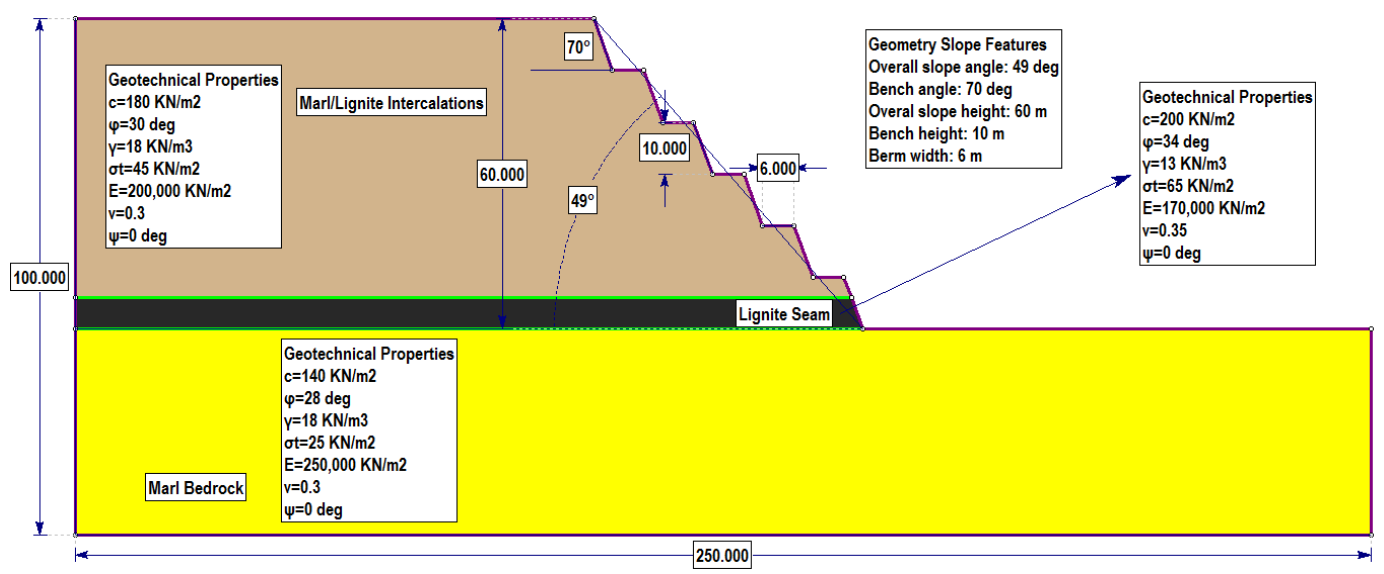

Figure 1 - Typical cross section of lignite mining slope.

\subsection{Modelling Assumptions}

The basic assumption in the present work is that slope failure is driven by the existence of excessive shear stresses within the soil mass, rather than on discontinuities or other weak planes. This was considered a reasonable assumption based on the geotechnical conditions of the problem (soil and/or soft rock conditions) and on the experience from the field. Hence, continuum mechanics based methods, such as FEM and FDM, were considered appropriate for this effort. The cross section that was numerically analyzed is illustrated in Figure 1. It shall be noted that due to the minor thickness of the thin lignite lenses within the overburden marl mass (typically less than $0.5 \mathrm{~m}$ ), it was considered more reasonable to model the upper layer (marl / lignite intercalations) as one homogeneous layer with approximately intermediate (between the primary formations of the marl and the lignite) engineering properties.

Overall, the cross section (Fig. 1) is considered representative of the slope under-examination. In addition, the geometry of the slope and the lithological boundaries are considered constant in the out of plane direction, therefore 2D plane strain conditions are used as a reasonable assumption. Imposed boundary conditions included horizontal fixities $\left(u_{x}=0\right)$ on the vertical boundaries and full fixities $\left(\mathrm{u}_{\mathrm{x}}=\mathrm{u}_{\mathrm{y}}=0\right)$ on the bottom boundary. An elastic - perfectly plastic Mohr - Coulomb constitutive model was used for the simulation of the geological materials (see Fig. 1 for values of corresponding parameters). At this stage of the research, groundwater table was not included in the simulations, so analyses were performed for long term (drained) conditions. Generally, geometric features, material properties, boundary conditions and other characteristics related to the models, were all taken identical in the three software analyses, in order to facilitate the comparative purpose of the work. Last but not least, about the lateral (left and right) and bottom extent of the model, their choice was 
based on the belief that they were adequate (i.e. large enough) for preliminary simulation purposes, in the sense that they were not expected to influence the analyses' outcomes.

\subsection{The Shear Strength Reduction (SSR) Technique}

As already mentioned in the introduction, analyses were performed using the so-called shear strength reduction technique (sometimes, it is mentioned in the literature as a method, however in the authors' opinion this is an exaggeration and should be avoided). Over the last 10-15 years, this technique (herein referred to as SSR technique) has become an integral part of most geotechnical numerical software packages. Through this technique, a safety factor is computed and a corresponding failure surface within the soil mass is exposed (Dawson et al., 1999). The SSR technique has been widely used in open pit slope stability studies. This is so because it includes all the benefits of limit equilibrium analyses (for example, among other things, it also results on a safety factor, which - for the vast majority of practitioners - remains the most familiar concept that desrcibes stability) and at the same time it allows the user to assess slope displacements that are often critical in the evaluation of open pit stability (Hoek, 2009). In the SSR type of analysis, safety factors are calculated by progressively reducing the shear strength of the material in order to mobilize the slope to the verge of failure, i.e. until non-convergence occurs within a specified number of iterations and tolerance. The ratio of the soil's actual shear strength to the reduced shear strength is the resulting safety factor. If $\varphi^{\prime}-c^{\prime}$ and $\varphi^{*}-c^{*}$ are the actual and the reduced parameters, respectively, then the SF can be written as (Matsui and San, 1992; Dawson et al., 1999):

$$
\mathrm{SF}=\frac{\tan \phi^{\circ}}{\tan \phi^{*}}=\frac{\mathrm{c}^{\sigma}}{\mathrm{c}^{*}}
$$

It shall be noted here that as far as the combination of constitutive models with the SSR technique is concerned, the use of a Mohr - Coulomb constitutive model is a prerequisite (instead of other, more advanced models) in the SSR features of the three software packages that were used. This is so because stress-dependent stiffness behavior and hardening effects (that would be captured by more advanced models) are usually excluded in this type of analyses.

\section{Results - Discussion}

In the context of the present work, three different but relevant to each other features are being sought through the analyses. These are the potential slip surfaces of the open pit slope, illustrated by means of shear strain localization, the kinetics of the collapse mechanism, illustrated through the displacement vectors distribution, and the "failure" extent, illustrated through plastic regions propagation.

\subsection{Slip Surface (Shear Strain Localization)}

The potential slip surfaces of the examined model are discussed in terms of shear strain localization (Fig. 2). In other words, it is considered that the concentration of maximum shear strains in the model at the time of collapse, corresponds to the developing failure surface of the slope.

As far as FLAC is concerned, shear strain rates contours identify regions where shear strain localizes. Shear localization bands or "shear bands" that develop in the model during calculation correspond to failure surfaces. Contours of the shear strain rates are shown in Figure 2a, on which maximum values are observed in the foot of the slope and at the vertical tensile crack behind the crest of the slope. Concerning the slip surface developed, this seems to be a typical circular surface commonly observed in this type of problems. In Plaxis, the total deviatoric shear strain contours may be used for the representation of the critical slip surface. In this case, shear strain localization and the global slip surface of the mining slope is shown in Figure $2 b$. Last, the slope failure surface generated by Phase2, is shown in Figure 2c by means of maximum shear strain contours. Again the maximum 
accumulation of shear deformations seems to be developed in the foot of the slope, however the spreading of the shear band in Phase 2 is quite larger compared to FLAC and Plaxis.
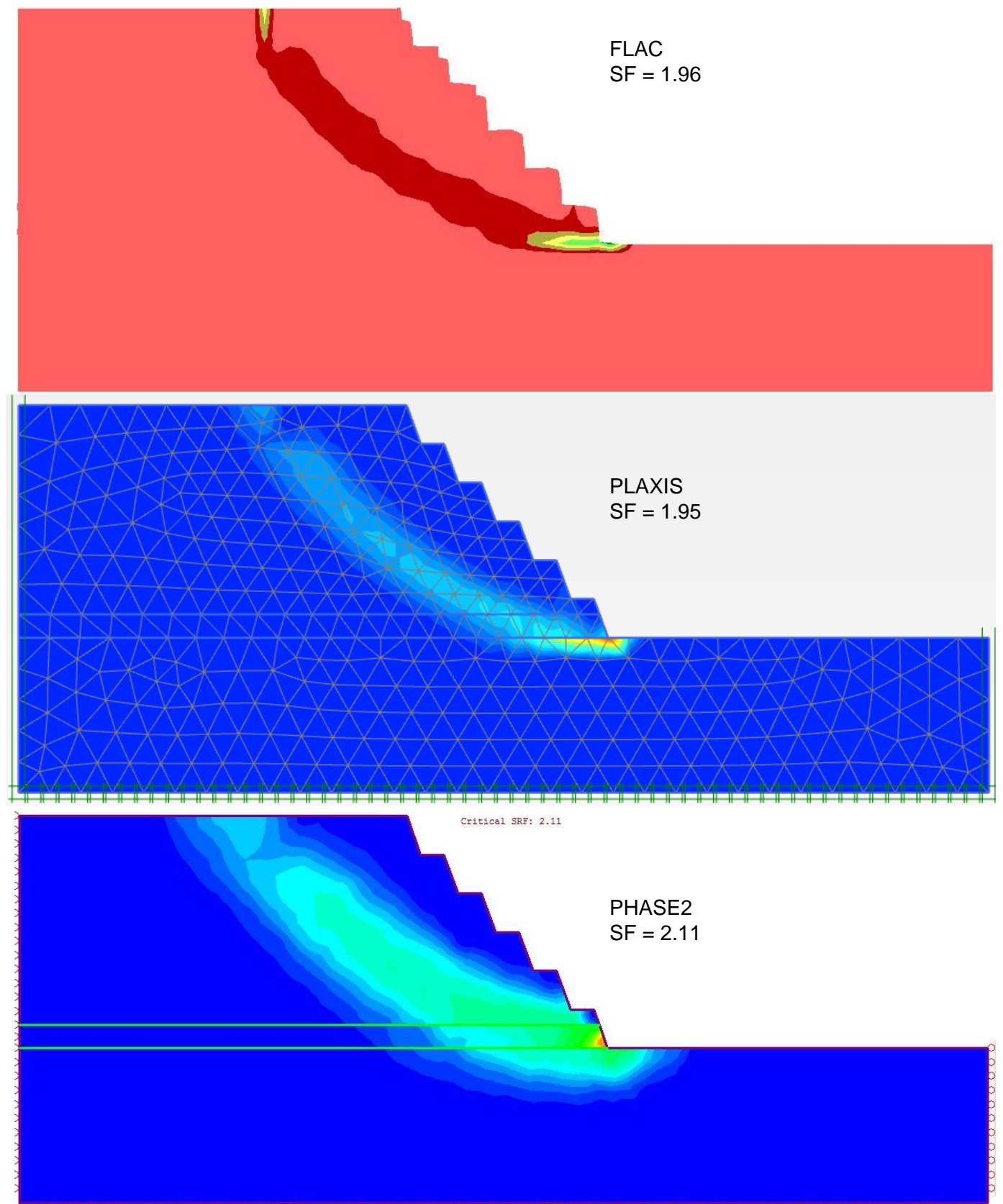

Figure 2 - Shear strain localization.

Overall, it can be said that in terms of shear strain concentrations and the indicated sliding surfaces, a good qualitative agreement occurs between FLAC and Plaxis. The mutual element between these two software, is the confined magnitude of shear strain concentration, which outlines a fine and distinct slip surface, while the respective shear strain localization and shear band in Phase2 is more extended and coarse. Finally, it is worth mentioning that among the three, FLAC seemed to be able to generate a clear vertical tensile crack behind the slope crest. 


\subsubsection{Safety Factors}

In terms of SF, all three software packages ended up with similar values: FLAC and Plaxis resulted in identical values ( $\mathrm{SF}=1.96$ and $\mathrm{SF}=1.95$, respectively), while Phase2 resulted in a slightly higher value (2.11). For the cross section under examination, this difference (between FLAC/Plaxis and Phase2) is considered negligible, given that the slope is apparently away from limit states of failure (ultimate limit states). Therefore, a difference between 1.95 and 2.11 probably would not make any difference in the design of the slope. However, this could not be the case in slopes close to the ultimate limit states (SF around 1). In such cases small differences in the resulting SFs (in the order of 0.1 to 0.2 ) would have raised further discussion.

\subsection{Kinetics of Collapse Mechanism (Displacement Vectors Distribution)}

Generally, the kinetics and the collapse mechanism of either potential (for safety precautions) or already-existing (for back analysis purposes) failures, provide useful information to the engineer. In the present work, kinetics and collapse mechanisms are discussed by means of vectors that describe the slope motion in the plane at the time of collapse. These are the velocity vectors, the incremental displacement vectors and the total displacement vectors, as used respectively in FLAC, Plaxis and Phase2 (Fig. 3). It shall be noted that although the direction and the size of the displacement/velocity vectors has no quantitative meaning on a SSR type of analysis, however they provide a good qualitative sense of the developing failure mechanism, since they reflect the physical pattern of the motion at the edge of collapse. As shown in Figure 3, in all three cases, the direction of displacement/velocity vectors reflect the developing rotational failure mode, which is typical on slopes on soils and/or soft rock. As mentioned earlier, in such conditions (where ground can be realistically simulated as a continuous medium), failure depends rather on the shear strength of the material than on the shear strength of the discontinuities. So, it can be said that the representation of the slope motion in failure is practically identical (qualitatively speaking) for all three different software packages.

\subsection{Failure Extent (Plastic Regions Propagation)}

Plastic regions propagation demonstrates the areas on which the yield criterion (Mohr - Coulomb in the present study) is satisfied. However, plastic regions do not necessarily coincide with the developing shear bands and do not always reflect the sliding surface of the slope. They usually occupy larger areas in numerical models than the shear strain localization. In fact, it is the combination of plastic failure extent with the shear strain concentrations that gives the critical developing sliding surface. Sometimes, the propagation of the plastic area in regions far away from the shear band either implies the possible presence of a secondary (and less significant) failure mechanism, or it might be simply an alarm of inadequate boundary conditions and model limits. In the light of the above, differences among the results of the three models regarding plastic regions within the slope mass and the corresponding failure extents were quite evident (Fig. 4). More specifically:

Figure 4a depicts the plasticity indicators on FLAC (circles denoting failures at tension, asterisks denoting failures at shear). The extent of the plastic points in this model is quite close to the area covered by the shear band (shear strain contours) described earlier. It is noted that the tension cutoff points behind the crest of the slope indicate the tensile cracks formed as a forerunner slope failure phenomenon. As shown in Figure 4a, plastic zone does not propagate within the marl material of the slope base (i.e. it is constrained in the lignite seam and the overburden layer). 


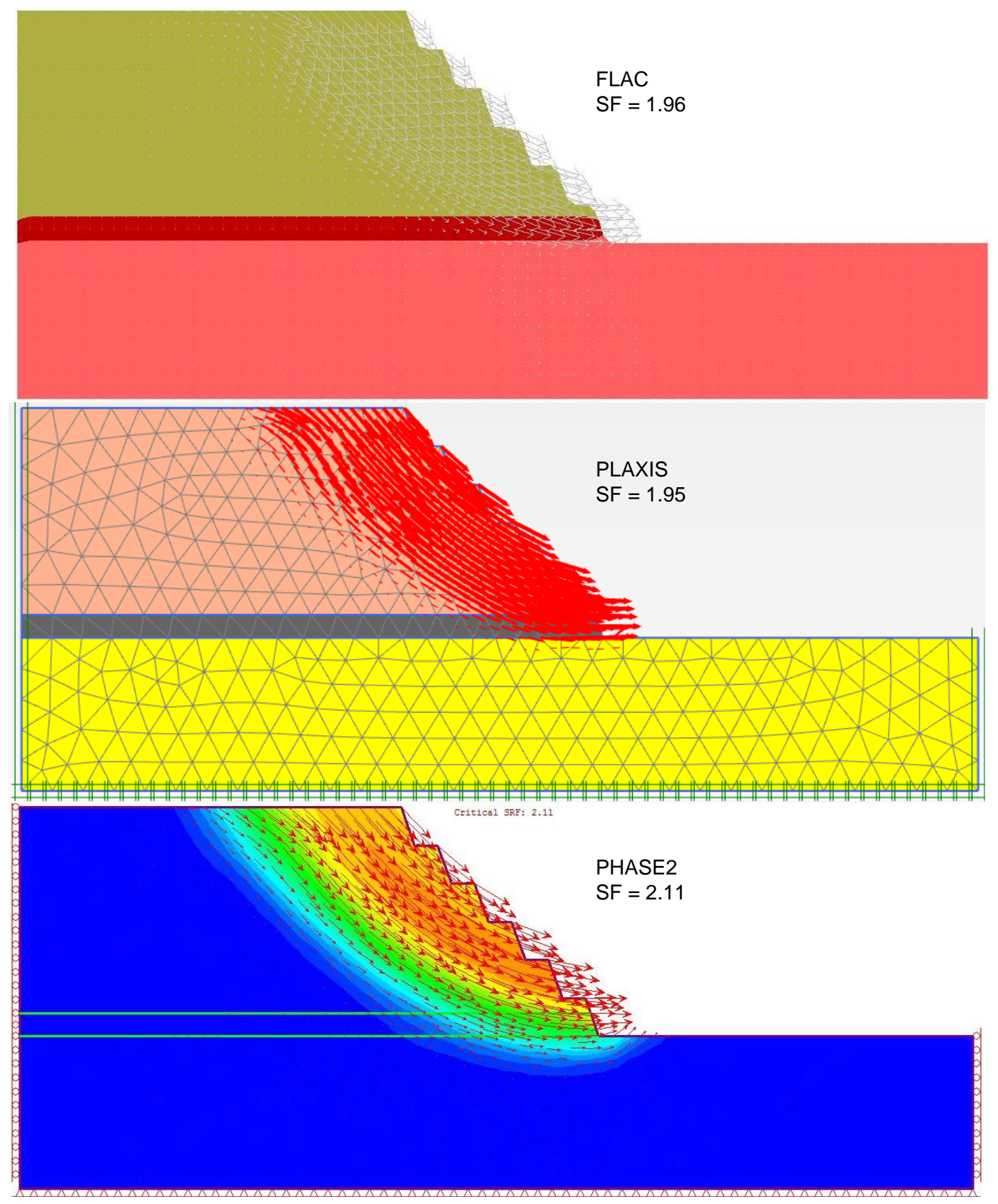

Figure 3 - Displacement vectors distribution.

On Plaxis (Fig. 4b, white squares denoting failure at tension, red squares denoting failures at shear), the area covered by plastic points is fairly more extended than the area covered by the shear band (see Fig. 2b), and more extended than the equivalent area covered by plastic points on FLAC (Fig. 4a). To be more specific, besides the major failure mechanism (represented by the plastic points mostly in the lignite and marl / lignite intercalations layers, but also slightly within the marl at the slope's base), a secondary cloud of plastic points extends towards the bottom boundary of the model, as a kind of a "deep-seated circular surface" in the underlying marl layer. The question that arises is whether this is indeed a secondary failure mechanism interrupted by the bottom horizontal boundary of the model, or it is simply a side effect of possibly inadequate bottom boundaries. Taking into 
account that something similar did not appear in the respective outputs of shear strain and total displacement distribution, but also taking into account field conditions, it was suspected that the original bottom boundaries of the problem might be inadequate.
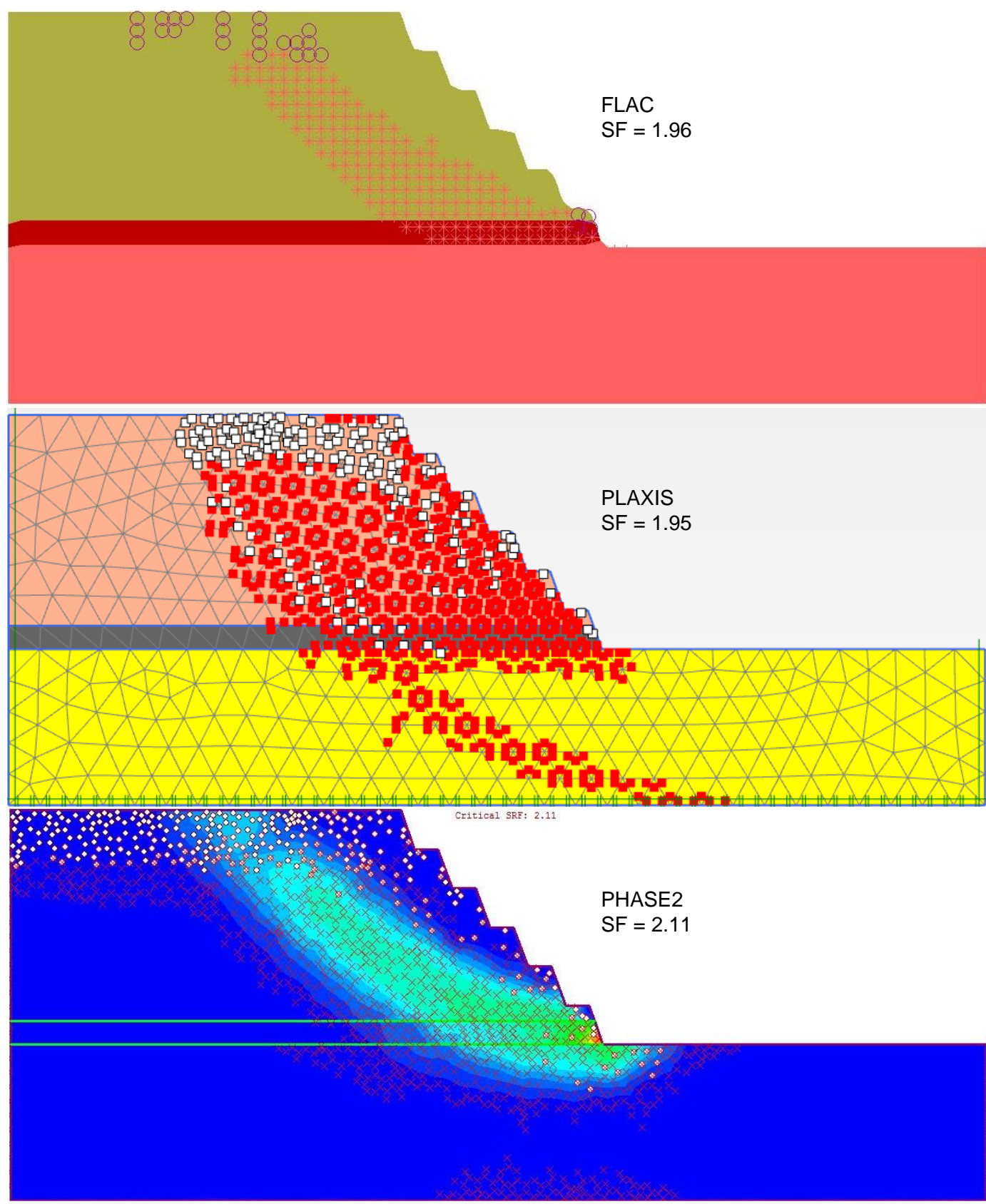

Figure 4 - Plastic regions propagation.

Even worst concerns were raised on Phase2 (Fig. 4c, white circles denoting failures at tension, asterisks denoting failures at shear), where yielded elements both in shear and tension are largely extended and greatly escape from the maximum shear strain concentrations of the model (Fig. 2c). More specifically, plastic points (asterisks) seem to extend well within the marl at the slope's base (compared to no intrusion at all of such points on FLAC and slight intrusion on Plaxis), while they 
also spread a lot within the slope mass itself towards the left vertical boundary of the problem. In addition, tensile failure reaches up to the left vertical boundary as well, and some shear failure points exist in the middle of the bottom boundary of the model. Taking into account the above (which do not coincide with the shear bands produced by the program), it is rather possible that the external boundaries on Phase 2 are insufficient for the solution scheme in terms of plastic/tension points. As far as the shear failure points in the bottom horizontal boundary, based on the authors' experience, this might be a bug-type of problem. This is so because it is a phenomenon that often occurs in the SSR technique on Phase2, on slope stability problems, regardless the model boundary conditions.

Due to the above observations on Plaxis and Phase2, a series of complementary analyses with extended bottom boundary limits was performed, in order to investigate further this issue. After a newly realized series of analyses in Plaxis, it was observed that the secondary cloud of plastic points (as well as the number of points failing at tension) was significantly reduced in the new extended model, compared to the original one. So, it was concluded that the previous results were rather an issue of bottom boundary limits. As for Phase2, in the new analysis, plastic points completely disappeared from the bottom boundary. However, the problem of "bizarre" locations of plastic points was shifted to the right and left vertical boundaries of the model, where both tensile (at the left boundary) and shear (at both the left and right boundaries) failure occurred more widely up to them in the extended model.

Based on all the above, it is fair to say that overall FLAC seems to be the most consistent in terms of agreement between the localization of maximum shear strains and the plasticity indicators development, since a good fit between them existed in the first place. In addition, FLAC did not require an extension of the bottom boundaries. On the other hand, plastic points both in Plaxis and Phase 2 models are quite extensive (in other words, the failure criterion is satisfied on these two more "easily" than on FLAC) and exceed significantly the contours of the maximum shear strains. For Plaxis, a second round of analysis with extended boundaries seems to improve the situation, while the same does not hold true for Phase2.

\section{Conclusions}

Slope stability of an open pit lignite mine was studied using three different numerical software packages: FLAC (finite difference), Plaxis (finite elements) and Phase2 (finite elements). The choice of continuum-mechanics based methods was justified by the nature of the involved geologic strata (soils and soft rocks). The baseline cross section was typical of a private mine excavated with noncontinuous extracting methods in Greece. Analyses were performed in drained, plane strain, conditions. Elastic - perfectly plastic Mohr - Coulomb constitutive models were used for simulating the mechanical behavior of the involved strata. The shear strength reduction technique was used as the analysis scheme. Based on the results of the study, all three programs demonstrated a good agreement in the determination of safety factors and the kinetics of collapse mechanisms (identification through displacement or velocity vectors). Critical sliding surfaces, identified by shear strains localizations, were found to be similar on FLAC and Plaxis, but somehow more extended onPhase2. Last but not least, noteworthy differences appeared among the three programs in the development of plastic regions (failure in shear) and of regions failing in tension. With respect to these, FLAC seemed to obtain the more realistic results and to be the most consistent in terms of agreement between the localization of maximum shear strains and the plasticity indicators development. The other two programs, either required more extended limits in order to achieve consistency between maximum shear strains and plasticity indicators, or insisted on producing large (non-realistic) areas of plastic failures. Overall, it can be said that all three programs may address the examined problem satisfactorily. However, future research work should investigate further the issue of plastic regions propagation. In addition, future work should include normal plastic analyses (without the SSR technique), in order to be able to simulate excavation sequences and, given that more elaborate laboratory data becomes available, use of more advanced constitutive models. 


\section{Acknowledgements}

This work has been funded by the European Commission through the Research Fund for Coal and Steel (Contracts RFCR-CT-2012-00003 COMEX and RFCR-CT-2015-00001 SLOPES). Financial assistance by the European Commission is greatly appreciated.

\section{References}

Agioutantis, Z., Stiakakis, E., Kalogirou, I. and Kourkoutis, S., 2006. An investigation of the stability and deformations of a deep open pit lignite mine using finite elements analysis, Mining Information and Analytical Bulletin, 10, 123-130.

Dawson, E., Roth, W. and Drescher, A., 1999. Slope Stability Analysis by Strength Reduction, Géotechnique, 49(6), 835-840.

Duncan, J.M., 1996. State of the art: limit equilibrium and finite-element analysis of slopes, Journal of Geotechnical Engineering, 122(7), 577-596.

Duncan, J.M. and Wright, S.G., 2005. Soil Strength and Slope Stability, Wiley \& Sons.

Euracoal, 2013. Coal Industry across Europe, $5^{\text {th }}$ ed., European Association for Coal and Lignite.

Griffiths, D. and Lane, P., 1999. Slope Stability Analysis by Finite Elements, Géotechnique, 49(3), 387-403.

Hoek, E., 2009. Fundamentals of Slope Design, Keynote address, Slope Stability 2009, Santiago, Chile, 9-11 November 2009.

Hustrulid, W., McCarter, M. and Van Zyl, D., 2001. Slope Stability in Surface Mining, Society for Mining, Metallurgy \& Exploration (SME).

Kavadas, M., Agioutanis, Z., Schilizzi, P. and Steiakakis, C., 2013. Stability and movements of open pit lignite mines in Northern Greece, Proccedings of the $18^{\text {th }}$ International Conference on Soil Mechanics and Geotechnical Engineering, Paris, 2193-2196.

Kliche, C.A., 2011. Slope Stability. In: SME Mining Engineering Handbook, Vol. 1, Chapter 8.3, In: Darling, P., ed., $3^{\text {rd }}$ ed., Society for Mining, Metallurgy \& Exploration (SME), 495-525.

Koukouzas, C. and Koukouzas, N., 2000. Classification of the Greek lignite deposits according to the United Nations International Framework classification for reserves/resources, Mineral Wealth, October-December 2000, 117, 19-36.

Leonardos, M. and Terezopoulos, N., 2003. Rim slope failure mechanism in the Greek deep lignite mines - a case study, Transactions - Institution of Mining \& Metallurgy, 112, 197-204.

Matsui, T. and San, K.C., 1992. Finite element slope stability analysis by shear strength reduction technique, Soils and Foundations, 32(1), 59-70.

Read, J. and Stacey, P., 2009. Guidelines for Open Pit Slope Design, CSIRO Publishing, Australia.

Steffen, O.K.H., Contreras, L.F., Terbrugge, P.J. and Venter, J., 2008. A Risk Evaluation Approach for Pit Slope Design, Proceedings, 42nd US Rock Mechanics Symposium and 2nd U.S.Canada Rock Mechanics Symposium, San Francisco, CA.

Steiakakis, E. and Agioutantis, Z., 2010. A kinetic behavior model at a surface lignite mine, based on geotechnical investigation, Simulation Modelling Practice and Theory, 18, 558-573.

Zevgolis, I.E., Koukouzas, N. and Schilizzi, P., 2015. Deformations and stability of a surface lignite mine - a challenging case study, Proceedings, AIMS 2015, $5^{\text {th }}$ International Symposium on Mineral Resources and Mine Development, Aachen, Germany. 